\title{
Impact of Active Surface Area on Performance and Reliability of Tri-gate FinFET
}

\author{
Yi-Lin Yang, ${ }^{1 *}$ Chiao-Feng Chuang, ${ }^{2}$ Chih-Jui Lai, ${ }^{1}$ Wenqi Zhang, ${ }^{2}$ Yun-Hsuan Hsu, ${ }^{2}$ \\ Chia-Jung Tsai, ${ }^{2}$ Wei-De Lin, ${ }^{2}$ Meng-Yen Lin, ${ }^{2}$ and Wen-Kuan Yeh $^{2,3}$ \\ ${ }^{1}$ Department of Electronic Engineering, National Kaohsiung Normal University, \\ No. 62, Shenjhong Rd., Yanchao District, Kaohsiung 824, Taiwan \\ ${ }^{2}$ Department of Electrical Engineering, National University of Kaohsiung, \\ No. 700, Kaohsiung University Rd., Nanzih District, Kaohsiung 811, Taiwan \\ ${ }^{3}$ National Nano Device Laboratories (NDL), National Applied Research Laboratories, \\ No. 26, Prosperity Road I, Hsinchu Science Park, Hsinchu 300, Taiwan
}

(Received Novemver 29, 2018; accepted May 8, 2019)

Keywords: active surface area (SA), contact etch stop layer (CESL), FinFET

In this work, a contact etch stop layer (CESL) was found to cause tensile stress above the gate of FinFET devices, and the top tensile stress introduced compressive stress in the channel. With increasing active surface area (SA), a higher compressive stress was observed. The effect of compressive stress became more evident, resulting in a lower current but a higher reliability for nFinFET devices.

\section{Introduction}

FinFETs are considered a promising candidate for device scaling that surpasses the framework of traditional planar transistors and has a better gate controlling capability, repressing short-channel effects (SCEs) and hot carrier effects (HCEs), and improving the subthreshold swing (SS). ${ }^{(1-5)}$ Even though fin-based structures may be superior electrically, they are less robust than planar structures mechanically, which may give rise to some unexpected failure mechanisms. ${ }^{(6)}$ Prior studies indicate that mechanical stress shows a great impact on the electrical behavior. In traditional planar MOSFETs, thermal annealing is considered to expand the filler in shallow trenches and squeeze it; thus, the channel region is subjected to a compressive stress. ${ }^{(7)}$ A typical fin bending may lead to electrical or physical failure. Its leaning angle forms an asymmetric depletion area and makes the leakage current larger than expected. ${ }^{(8)}$ On the other hand, tensile and compressive stresses, which are two components of mechanical stress, will respectively improve electron and hole mobilities. ${ }^{(9,10)}$ Stresses are intentionally introduced into the device channel by means of a silicon-carbon (SiC) source and drain (S/D), a strained cap layer, shallow trench insulation (STI), and so forth during the transistor fabrication process. In addition, lattice-mismatched $\mathrm{SiC}$ regions also induce stress in the Si channel along the width (transverse) and vertical directions. ${ }^{(11)}$ In fact, with the

*Corresponding author: e-mail: t3550@nknu.edu.tw

https://doi.org/10.18494/SAM.2019.2209 
continuous shrinking of the feature size, the STI stress effect becomes significant. ${ }^{(12)}$ However, this behavior is much different from those of FinFETs and MOSFETs. In this work, the electrical and reliability analyses of tri-gate FinFETs affected by different active surface areas (SAs) were studied.

\section{Experimental Methods}

Figure 1 shows a TEM image of the tri-gate FinFET used in this study. The channel length, fin width, and fin height of the device were 16, 10, and $42 \mathrm{~nm}$, respectively. The surface orientation of the fin sidewall is (110), while the channel direction is $<110>$. After FinFET device fabrication, SiN was covered as a contact etch stop layer (CESL). The CESL obtained by thermal CVD has tensile stress due to the manufacturing process. SA was defined as the distance from the gate to the edge of shallow trench isolation (STI), as shown in Fig. 2, and different SAs of $0.098,0.386$, and $3.842 \mu \mathrm{m}$ were utilized.

In this work, the electrical properties of FinFET devices were measured using a semiconductor parameter analyzer (Agilent-B1500A) at room temperature. During hot carrier injection (HCI) stress measurement, the drain and gate voltages were set as $V_{D}=V_{G}=1.6 \mathrm{~V}$ with the grounded source and body. $I-V$ curves were measured at certain stress time intervals.

\section{Results and Discussion}

Figure 3 shows $I_{D}-V_{G}$ curves with different SAs. The extracted values of $V_{T H}$ and SS are respectively shown in Figs. 4(a) and 4(b). It was observed that $V_{T H}$ increases with SA, while SS is similar to the variation in SA. However, a reduction in drain current was observed with increasing SA. All samples are fabricated under the same conditions except for SA. As a result, the increase in $V_{T H}$ and the reduction in $I_{D}$ might be due to the influence of SA. On the other hand, similar SS values also indicate that different SAs did not affect the interface quality of devices.

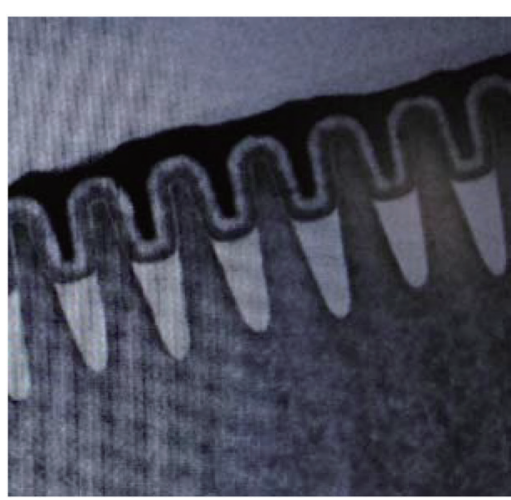

Fig. 1. (Color online) TEM image of the tri-gate FinFET.

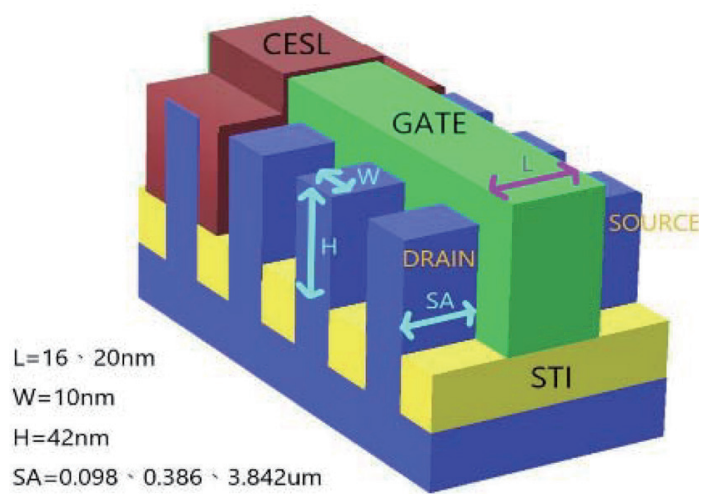

Fig. 2. (Color online) Structure and definition of SAs specifications for FinFET. 


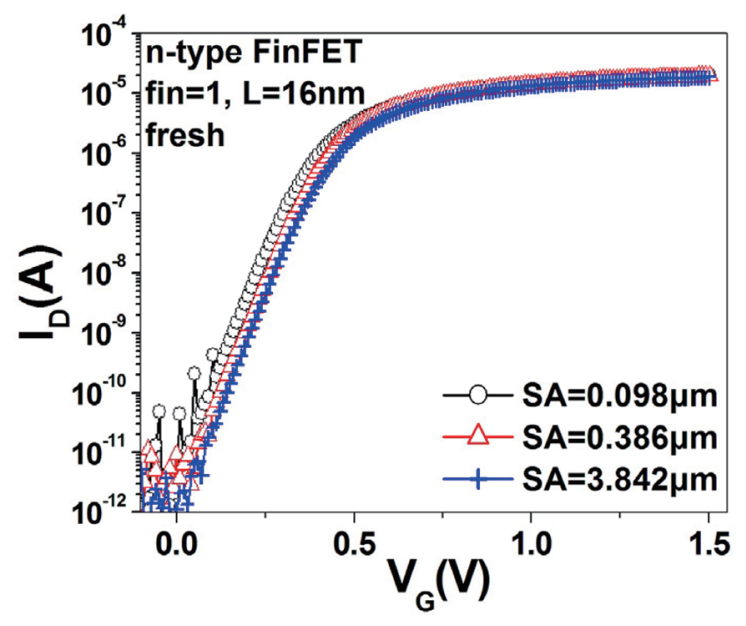

Fig. 3. (Color online) $I_{D}-V_{G}$ curves of different SA specifications.

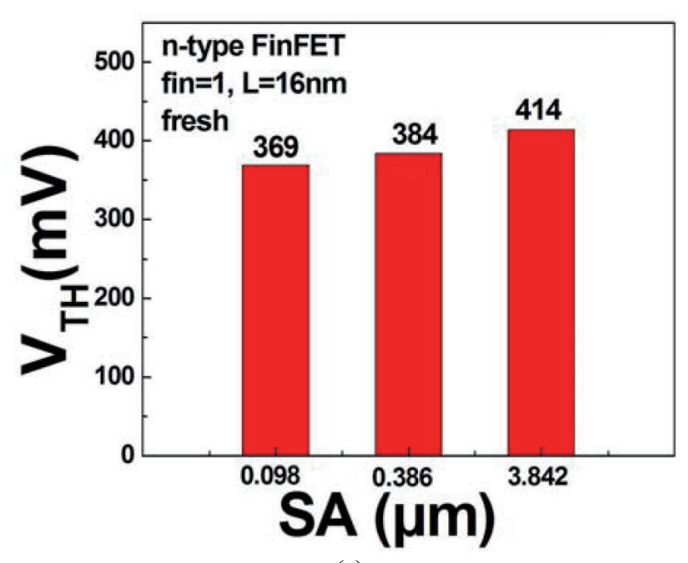

(a)

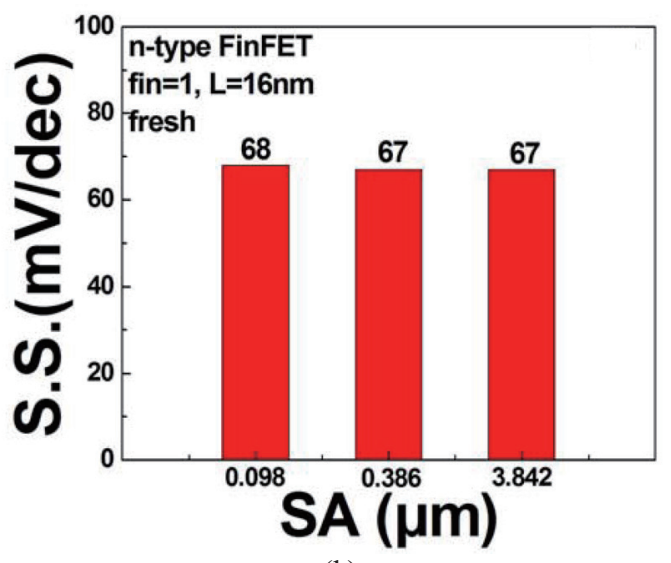

(b)

Fig. 4. (Color online) Extracted values of (a) $V_{T H}$ and (b) SS with different SA specifications.

HCI was then utilized to investigate the reliability of FinFET devices with different SAs. The $I_{D}-V_{G}$ curves obtained before and after 6000s HCI are plotted in Fig. 5. The positive $V_{T H}$ shift during HCI stress indicates that the injected electrons were trapped in the gate dielectric layer. On the other hand, SS degradation reveals that impact ionization, which induces the generation of the interface state, occurs in FinFET devices during HCI stress. ${ }^{(2)}$ It was observed that the FinFET device with a smaller SA shows a more severe degradation of $V_{T H}$ and the subthreshold slope. The $V_{T H}$ shift and SS variation versus stress time were extracted and are shown in Figs. 6(a) and 6(b), respectively. A $V_{T H}$ shift of $441 \mathrm{mV}$ was found in the device with $\mathrm{SA}=0.098 \mu \mathrm{m}$, while a $V_{T H}$ shift of $327 \mathrm{mV}$ was observed in the device with $\mathrm{SA}=3.842 \mu \mathrm{m}$. On the other hand, the increase in SS variation was determined to be $69 \mathrm{mV}$ for the device with $\mathrm{SA}=0.098 \mu \mathrm{m}$ and $42 \mathrm{mV}$ for the device with $\mathrm{SA}=3.842 \mu \mathrm{m}$. It was observed that the FinFET device with a larger SA shows a higher reliability. 


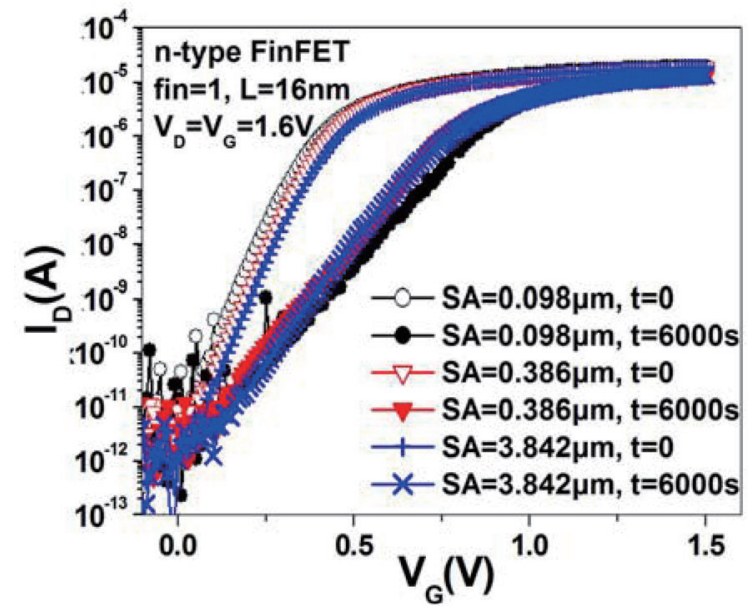

Fig. 5. (Color online) $I_{D}-V_{G}$ curves with different SAs after HCI stress for $6000 \mathrm{~s}$.

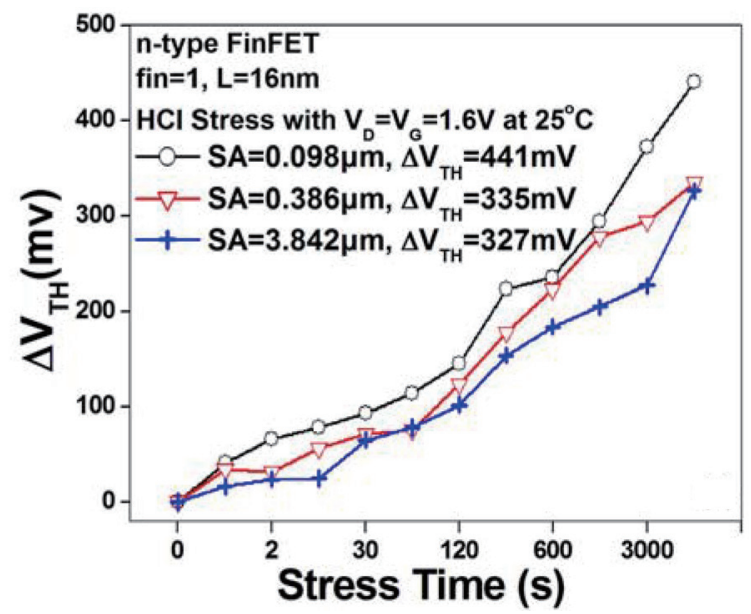

(a)

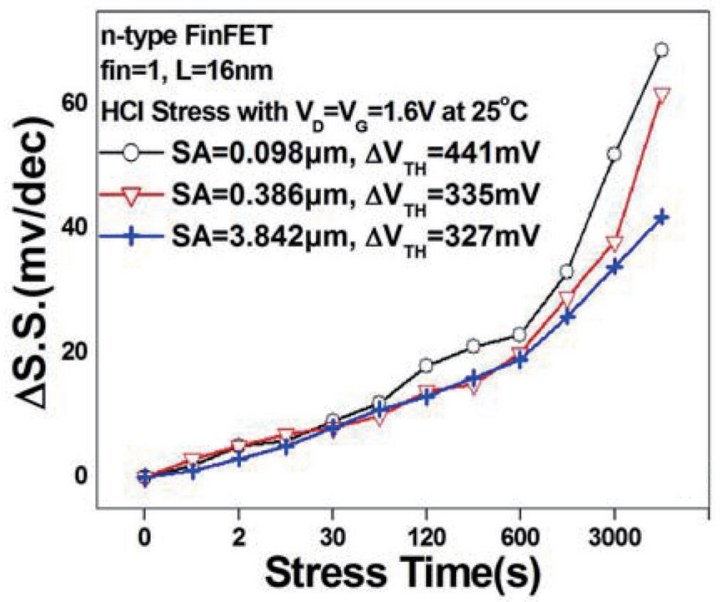

(b)

Fig. 6. (Color online) Extracted values of (a) $V_{T H}$ shift and (b) subthreshold slope variation versus stress time with different SA specifications.

To examine the effect of different SA specifications, the schematic diagram of SA-induced channel stress components is shown in Fig. 7. The FinFET device used in this work was covered with $\mathrm{SiN}$ deposited in a furnace, thus resulting in tensile stress above the gate, which is expressed as $\mathrm{T}$ in Fig. 7. The tensile stress above the gate and $\mathrm{S} / \mathrm{D}$ is expected to introduce a compressive stress to the channel, which is expressed as $\mathrm{C}$ in the diagram. As a result, a compressive stress to the channel is expected as the influence of SA. With increasing SA, the larger area covered by the CESL is expected to cause a higher tensile stress above the gate. The larger bending above the gate would lead to a higher compressive stress to the channel.

The reliability improvement of FinFET devices with the increase in SA can be explained as follows. With the increase in SA, the higher compressive stress reduces the electron mobility in the channel of nFinFETs. The reduced electron channel mobility results in a weaker 


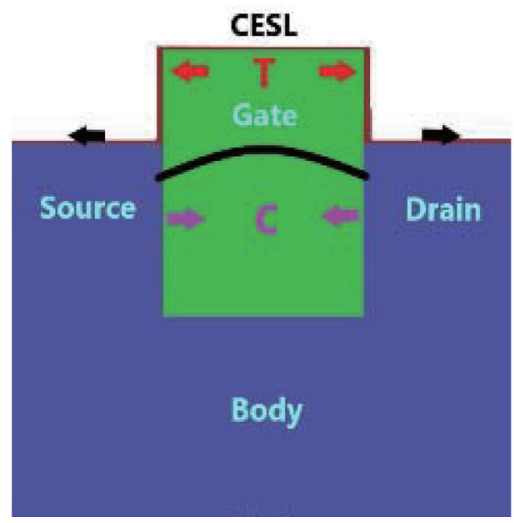

Fig. 7. (Color online) SA-induced channel stress and bending mechanism for FinFET devices.

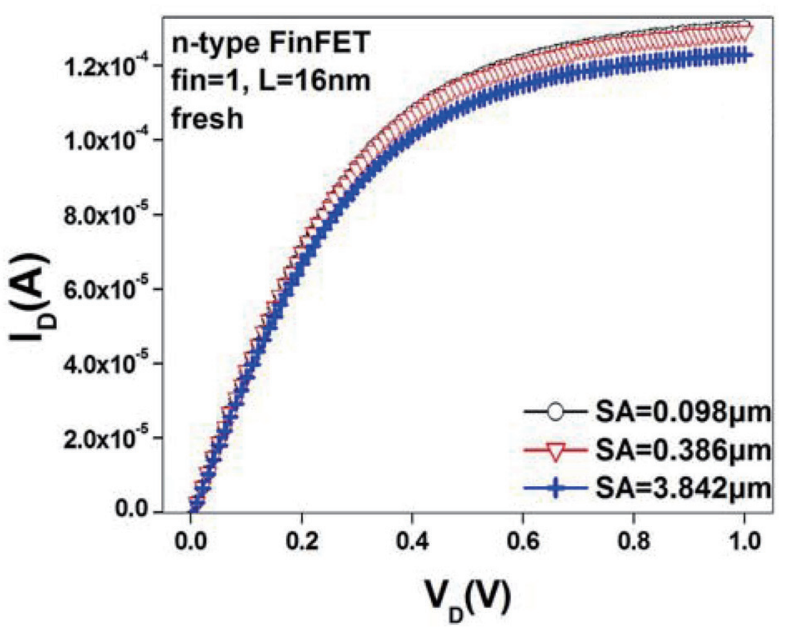

(a)

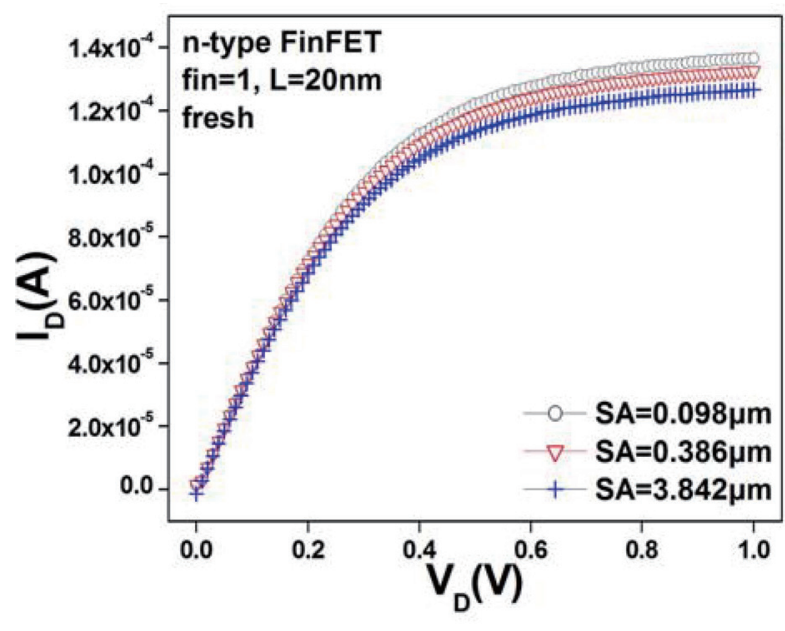

(b)

Fig. 8. (Color online) $I_{D}-V_{D}$ curves of FinFETs with different SAs at (a) $L=16$ and (b) $20 \mathrm{~nm}$.

ionization impact during HCI stress, which inhibits the generation of the interface state and thus suppresses subthreshold slope degradation. On the other hand, fewer electrons are injected and trapped into the gate dielectric layer owing to the slower channel electrons, decreasing the positive shift of $V_{T H}$ under HCI stress.

To verify the effect of compressive stress under different SA specifications, FinFET devices with different channel lengths were studied in this work, and the $I_{D}-V_{D}$ curves obtained at $L=16$ and $20 \mathrm{~nm}$ are respectively shown in Figs. 8(a) and 8(b). A reduced drain current was observed in the device with $\mathrm{SA}=3.842 \mu \mathrm{m}$ as compared with that obtained with $\mathrm{SA}=0.098 \mu \mathrm{m}$, and the degradation rates were determined to be 5.0 and 7.8\% for FinFET devices with $L=16$ and $20 \mathrm{~nm}$, respectively. These results could be explained by SA-induced compressive stress. For the longer channel device, the tensile stress provided by the CESL is enhanced by the larger area above the channel. The larger tensile stress above the gate introduces a higher compressive stress in the channel. A more severe drain current degradation is expected in the longer channel 
device owing to the higher compressive stress, and the higher compressive stress reduces the electron mobility more effectively. As a result, a more severe $I_{D}$ degradation for the longer channel with increasing SA could be explained.

\section{Conclusions}

N-type FinFET devices with different SAs were studied in this work. Compressive stress was found in the channel after CESL deposition. A large SA is considered to introduce a high compressive stress to the channel, thus reducing the channel electron mobility. As a result, a reduced device performance but an improved reliability is expected for FinFET devices with larger SAs.

\section{Acknowledgments}

This work was supported by the Ministry of Science and Technology under Contract of MOST 106-2221-E-390-018, and the authors would like to thank the staff of National Nano Device Laboratories (NDL) for their commendable support.

\section{References}

1 Y. Yang, W. Zhang, T. Yen, J. Hong, J. Wong, C. Ku, T. Wu, T. Wang, C. Li, B. Wu, S. Lin, and W. Yeh: Appl. Phys. Lett. 104 (2014) 2. https://doi.org/10.1063/1.4866437

2 W. Zhang, T. Wang, Y. Huang, T. Cheng, S. Chen, Y. Li, C. Hsu, C. Lai, W. Yeh, and Y. Yang: Microelectron. Reliab. 67 (2016) 89. https://doi.org/10.1016/j.microrel.2016.10.015

3 A. Paul, A. Bryant, T. B. Hook, C. C. Yeh, V. Kamineni, J. B. Johnson, N. Tripathi, T. Yamashita, G. Tsutsui, V. Basker, T. E. Standaert, J. Faltermeier, B. S. Haran, S. Kanakasabapathy, H. Bu, J. Chol, J. Iacoponil, and M. Khare: IEEE IEDM Tech. Dig. (2013) 361. https://doi.org/10.1109/IEDM.2013.6724625

4 P. Kerber, R. Kanj, and R. V. Joshi: IEEE Electron Dev. Lett. 34 (2013) 876. https://doi.org/10.1109/ LED.2013.2264620

5 W. Yeh, W. Zhang, Y. Yang, A. Dai, K. Wu, T. Chou, C. Lin, K. Gan, C. Shih, and P. Chen: IEEE Trans. Device Mater. Reliab. 16 (2016) 610. https://doi.org/10.1109/TDMR.2016.2612703

6 A. H. Gencer, D. Tsamados, and V. Moroz: Int. Conf. Simulation of Semiconductor Processes and Devices (SISPAD, 2013) 109. https://doi.org/10.1109/SISPAD.2013.6650586

7 B. C. Wang, S. L. Wu, and S. J. Chang: J. Vac. Soc. R.O.C. 24 (2011) 32. https://doi. org/10.29808\%2fJVSROC.201103.0006

8 C. Li, H. Zhao, and G. Mao: 2017 China Semiconductor Technology Int. Conf. https://doi.org/10.1109/ CSTIC.2017.7919800

9 T. Ghani, M. Armstrong, C. Auth, M. Bost, P. Charvat, G. Glass, T. Hoffmann, K. Johnson, C. Kenyon, J. Klaus, B. Mclntyre, K. Mistry, A. Murthy, J. Sandford, M. Silberstein, S. Sivakumar, P. Smith, K. Zawadzki, S. Thompson, and M. Bohr: IEDM Tech. Dig. (2003). https://doi.org/10.1109/IEDM.2003.1269442

10 F. Andrieu, T. Ernst, F. Lime, F. Rochette, K. Romanjek, S. Barraud, C. Ravit, F. Boeuf, M. Jurczak, M. Casse, O. Weber, L. Brevard, G. Reimbold, G. Ghibaudo, and S. Deleonibus: Proc. Symp. VLSI Technol. (2005) 176. https://doi.org/10.1109/.2005.1469257

11 W. Wang, S. T. Chang, J. Huang, S. H. Liao, and C. Y. Lin: IEEE Conf. Electron Devices and Solid-State Circuits (2007). https://doi.org/10.1109/EDSSC.2007.4450313

12 X.Fang, L. Sun, J. Liu, and H. Wang: IEEE Int. Symp. Integrated Circuits (2011). https://doi.org/10.1109/ ISICir.2011.6132011. 


\section{About the Authors}

Yi-Lin Yang was born in Taichung, Taiwan, on October 28, 1979. He received his B.S. degree in electronics engineering from National Chiao-Tung University in 2002, and his Ph.D. degree from the Graduate Institute of Electronics Engineering from National Taiwan University in 2009. He joined the faculty of the Department of Electronic Engineering, National Kaohsiung Normal University in 2010. His research interests focus on the high-k gate dielectrics, MOS devices, and nonvolatile memory.

Chiao-Feng Chuang received his B.S. degree in electrical engineering from National University of Kaohsiung, Taiwan, in 2015 and his M.S. degree from National University of Kaohsiung, Taiwan, in 2018.

Chih-Jui Lai received his B.S. degree in electrical engineering from National Kaohsiung Normal University, Taiwan, in 2016. He is currently a graduate student at National Kaohsiung Normal University.

Wenqi Zhang was born in Suzhou, Jiangsu, China, in 1982. She received her B.S. degree in materials chemistry from Nanjing University, Nanjing, China, in 2005, and her Ph.D. degree in materials physics and chemistry from Nanjing University, Nanjing, China, in 2011. In 20082009, she joined the Department of Electrical Engineering, Yale University, as a visiting student to investigate high-k dielectrics and nitride-based nonvolatile flash memory. She is currently a postdoctoral fellow in the Department of Electrical Engineering, National University of Kaohsiung, Taiwan, and is currently working on new-generation nanoscaled CMOSFETs.

Yun-Hsuan Hsu is an undergraduate student at National University of Kaohsiung.

Chia-Jung Tsai is an undergraduate student at National University of Kaohsiung.

Wei-De Lin received his B.S. degree in electrical engineering from National University of Kaohsiung, Taiwan, in 2016. He is currently a graduate student at National University of Kaohsiung.

Meng-Yen Lin received his B.S. degree in electrical engineering from National University of Kaohsiung, Taiwan, in 2016. He is currently a graduate student at National University of Kaohsiung.

Wen-Kuan Yeh was born in Hsin-Chu, Taiwan, in 1964. He received his B.S. degree in electronic engineering from Chung-Yuan Christian University, Chung-Li, Taiwan, in 1988, his M.S. degree in electrical engineering from National Cheng Kung University, Tainan, Taiwan, in 1990, and his Ph.D. degree in electronics engineering from National Chiao-Tung University, Hsin-Chu, Taiwan, in 1996. In 1989-1990, he joined the Taiwan Semiconductor Manufacturing 
Corporation (TSMC) Research and Development Division as an intern to research sub-um CMOSFETs. He also joined the United Microelectronic Corporation Technology \& Process Development Division as a research staff member to research and develop logic, embedded DRAM, SOI, and $90 \mathrm{~nm}$ transistor technological applications. He is currently a full professor of the Electrical Engineering Department and the Dean of Engineering in National University of Kaohsiung. He is also the Chair of the IEEE EDS Tainan Chapter. He has published 2 edited books, over 100 peer-reviewed papers, 3 book chapters, and over 80 patent applications. His recent work is in the field of nanoscaled CMOSFETs, SOI MOSFETs, and FinFETs. 